\title{
Splenic preservation versus splenectomy during laparoscopic distal pancreatectomy for benign and low-grade malignant pancreatic tumor: A propensity score matching analysis
}

\author{
Yoo-Seok YOON*
}

Department of Surgery, Seoul National University Bundang Hospital, Seoul National University College of Medicine, Seoul, Korea

Lecture: Previous studies showed the advantages of spleen preservation during laparoscopic distal pancreatectomy (LDP) such as preventing overwhelming postsplenectomy infection, and early infectious complications, maintaining immune surveillance, less postoperative pancreatic fistula, less postpancreatectomy diabetes, and less gastric ileus.

However, there were limitations of these results such as heterogenous population, and small number of patients. This study was aimed to compare clinical outcomes of patients with LDP with splenectomy (LDPS) and laparoscopic spleen preserving distal pancreatectomy (LSDP) using propensity score matching analysis from data of multi centers in Korea and Japan. The patients were identified who underwent LDPS and LSDP in Korea and Japan. Perioperative outcomes including postoperative infectious complication were compared between LDPS and LSDP before and after PSM. Matching variables were used such as nation, age, sex, BMI, ASA scores, underlying disease, previous abdominal surgery, preoperative pancreatitis, malignant disease and tumor locations.

LDPS and LSDP were classified in 1,728 (39.6\%), and 2,635 (60.4\%), respectively. In the mean age and body mass index were 56.3 and 23.4. And 1,166 (26.7\%) patients underwent previous abdominal surgery and tumor was located in body and tail in 2,041 (46.8\%) and 2,290 (52.5\%), respectively. Pancreas was transected at pancreas neck and tail level in 1,794 (41.1\%) and 2,521 (57.8\%), respectively. Splenic vessels were preserved in 1,244 (28.5\%) of 2,635 patients with LSDP. Two hundred patients (4.6\%) experienced conversion to open during surgery. Of patients, 1,303 (29.9\%) patients experienced postoperative complications. Clinically related postoperative pancreatic fistula (CR-POPF) and infectious complication rates were 693 (15.3\%) and 760 (17.4\%) patients, respectively.

After PSM, 1,594 patients were selected in each group. The patients with LSDP showed comparable overall complication (35.7 \% vs. $38.4 \%, p=0.094)$, however LSDP group showed lower infectious complications ( $15.2 \%$ vs. $19.1 \%, p=0.036)$, CR-POPF $(13.7 \%$ vs. $18.7 \%, p<$ $0.001)$, infected POPF (5.7\% vs. $9.7 \%, p<0.001)$, complicated fluid collection $(0.94 \%$ vs. $2.82 \%, p<0.001)$ compared with the patients with LDP.

The results of this study showed that LSDP was related with lower infectious complication rate including lower infected POPF, and lower complicated fluid collection compared with LDPS, and this result is helpful to decide splenic preservation in patients who needs LDP for pancreatic body or tail lesions. 